\title{
Non-Gaussian larval dispersal kernels in Gaussian ocean flows
}

\author{
Stephen M. Chiswell* \\ National Institute of Water and Atmospheric Research (NIWA), PO Box 14-901, Kilbirnie, Wellington, New Zealand
}

\begin{abstract}
Models of larval dispersal in which ocean currents are Gaussian-distributed provide analytical solutions for the dispersal kernel that are usually assumed to be Gaussian-distributed as well. However, such Gaussian solutions are only valid for a single value of the duration of the larval period. In reality, larvae have a settlement competency period that can range from minutes to $>1 \mathrm{yr}$. Dispersal kernels computed for larvae having a competency period of finite length become non-Gaussian, because later settlers contribute proportionately more to both tails of the kernels than early settlers. In species where the variability in larval duration is large, or mean flows are large compared to the variability, the dispersal kernels approach uniform rather than Gaussian distributions.
\end{abstract}

KEY WORDS: Larval dispersal - Spatial kernels · Connectivity

Resale or republication not permitted without written consent of the publisher

\section{INTRODUCTION}

A planktonic larval phase is instrumental in dispersal of most subtidal and intertidal species (e.g. Cowen \& Sponaugle 2009). The spatial distribution of settlement in larvae released from a given source is known as the 'dispersal kernel' (e.g. Aiken et al. 2007), or 'spatial kernel' (to distinguish it from the temporal kernel, i.e. the age distribution of settling larvae; e.g. Chiswell 2011). Knowledge of the spatial kernel is critical for the design of marine protected areas (e.g. Shanks et al. 2003) or in developing a response to harmful invasive species (e.g. Trakhtenbrot et al. 2005).

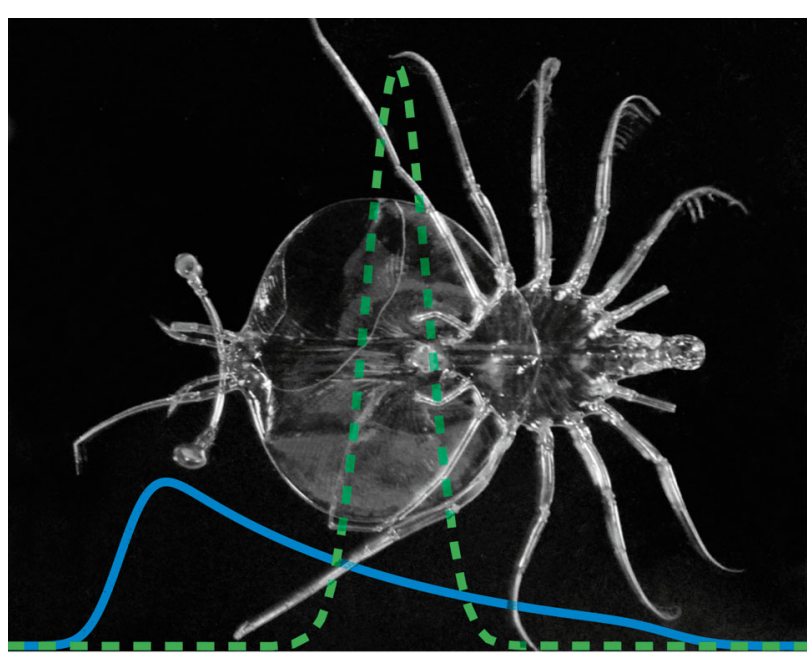

Late-stage phylosoma of spiny lobster Jasus sp. Larvae of this genus have the longest known competency period among invertebrate larvae (12 to 24 mo), leading to considerable variability in spatial scales of settlement.

Photo: Oddgeir Alvheim, IMR, Bergen, Norway

It is often assumed that ocean currents have a Gaussian distribution; in that case, for a single value of the larval period, solutions for the spatial kernel are well known, analytic, and also Gaussian (e.g. Largier 2003, Kinlan et al. 2005). Therefore, some authors fit Gaussian distributions to unresolved numerically simulated kernels (e.g. Mitarai et al. 2008). In reality, however, the larval period does not have a single value; instead, there is a competency period during which larvae can settle. The competency period can range from minutes to $>1 \mathrm{yr}$, depending on species and environmental conditions 
such as temperature, food or substrate availability (e.g. O'Connor et al. 2007). For example, larvae of the bryozoan Bugula neritina can settle from $5 \mathrm{~min}$ to $30 \mathrm{~h}$ after spawning (Shanks et al. 2003), whereas the competency period is from 30 to $60 \mathrm{~d}$ in sea urchins Evechinus chloroticus (Walker 1984), and 12 to $24 \mathrm{mo}$ in rock lobsters Jasus edwardsii (Booth 2002).

The present analysis shows that if there is a range in the duration of the larval period, the distribution of the spatial kernel is non-Gaussian, even when the distribution of ocean currents is Gaussian. When the range in larval period is short, a Gaussian approximation for the kernel may be adequate, but if the range is long or currents are strong, the kernel can become significantly nonGaussian.

\section{MODEL}

The solutions presented here are for one-dimensional flow, and consider the spatial distribution of settlement for larvae released from a point source into a Gaussian-distributed velocity field. Following Chiswell (2011), the ocean velocity field is described as containing a mean flow plus variability associated with an eddy field. This variability is parameterised in terms of an ocean eddy diffusivity, $K$.

The one-dimensional Lagrangian velocity, $u$ (i.e. the velocity seen by an individual larva), can be described as a function of time, $t$, as a Markov 1 random walk (e.g. Rupolo 2007) in the presence of a mean flow:

$$
u(t)=u_{0}+u^{\prime}(t)
$$

where $u_{0}$ is the mean velocity, and $u^{\prime}$ is the randomwalk eddy variability. This eddy variability is assumed to be Gaussian-distributed, has a standard deviation $u_{1}$, and a Lagrangian decorrelation timescale $T_{\mathrm{L}}$ (a measure of the spectrum of the eddy processes which lead to dispersal), so that $K$ is the product of this timescale multiplied by the variance of velocity (e.g. Rupolo 2007):

$$
K=u_{1}^{2} T_{\mathrm{L}}
$$

The ocean can thus be described to first order by its mean velocity and eddy diffusivity.

For a single value of larval period, $\tau$, the distribution of displacement at settlement, i.e. the spatial kernel $\left.S(x)\right|_{\tau}$ is expected to be Gaussian (Largier 2003, and references therein):

$$
\left.S(x)\right|_{\tau}=\left(\frac{1}{\sqrt{2 \pi} \sigma_{x}}\right)^{\frac{-(x-\bar{x})^{2}}{2 \sigma_{X}{ }^{2}}}
$$

where $x$ is displacement, and $\bar{x}$ and $\sigma_{x}$ are the mean and SD of displacement, respectively. The integral of the spatial kernel is 1 , so that it can be regarded as a probability density function of larval settlement.

The mean displacement is the product of mean velocity and larval period:

$$
\bar{x}=u_{0} \tau
$$

The variance in the distribution increases linearly in time, proportional to eddy diffusivity and larval period:

$$
\sigma_{x}^{2}=2 K \tau
$$

Eqs. (3 to 5) thus describe the spatial kernel for a larval period $\tau$ in an ocean defined by $u_{0}$ and $K$.

When the larval period ranges from $T_{1}$ (earliest settlement) to $T_{2}$ (latest settlement), the dispersal kernel is the sum of kernels over the range $T_{1}$ to $T_{2}$. By substituting Eq. (5) into Eq. (3) and integrating, the unnormalised dispersal kernel can be written as

$$
\left.S(x)\right|_{T_{1}: T_{2}}=\int_{T_{1}}^{T_{2}} \gamma(\tau) \frac{1}{\sqrt{4 \pi K \tau}} \exp \frac{-\left(x-u_{0} \tau\right)^{2}}{4 K \tau} \mathrm{d} \tau
$$

where the settling rate $\gamma(\tau)$ takes into account both the natural distribution in larval periods and mortality.

\section{RESULTS}

Eq. (6) cannot be solved analytically, but can be integrated numerically, and Fig. 1 illustrates the solution for hypothetical larval periods that span 2 to $12 \mathrm{~d}$ in an ocean where $u_{0}=0.1 \mathrm{~m} \mathrm{~s}^{-1}, u_{1}=0.03 \mathrm{~m}$ $\mathrm{s}^{-1}$, and $T_{\mathrm{L}}=4 \mathrm{~d}$. These values are realistic for many parts of the continental shelf (e.g. the Southland Current, New Zealand; Chiswell 1996) and correspond to an ocean having $K \sim 300 \mathrm{~m}^{2} \mathrm{~s}^{-1}$. Spatial kernels $\left.S(x)\right|_{2}$ and $\left.S(x)\right|_{12}$ were calculated for the minimum and maximum of the larval period, respectively, along with 2 estimates of the kernel calculated over the full period, $\left.S(x)\right|_{2: 12}$. The estimates of $\left.S(x)\right|_{2: 12}$ were calculated for both uniform settling rate $\left(\gamma(t)=\gamma_{0}\right)$ and for a rate largely determined by exponential mortality $\left(\gamma(t)=\gamma_{0} \exp \left(-t^{2} / \mathrm{m}\right)\right)$, where the last arrivals settle at $10 \%$ of the rate of the first arrivals (i.e. $\left.\mathrm{m}=\left(T_{2}-T_{1}\right) / \ln (10)\right)$. Fig. 1 illustrates that later settlers contribute proportionately more settlement at both tails of the kernel than do early settlers. The net effect is that $\left.S(\mathrm{x})\right|_{2: 12}$ is positively skewed. Adding mortality skews the kernel further toward earlier arrivals, but even with the severe mortality illustrated here, the kernels are significantly non-Gaussian. 


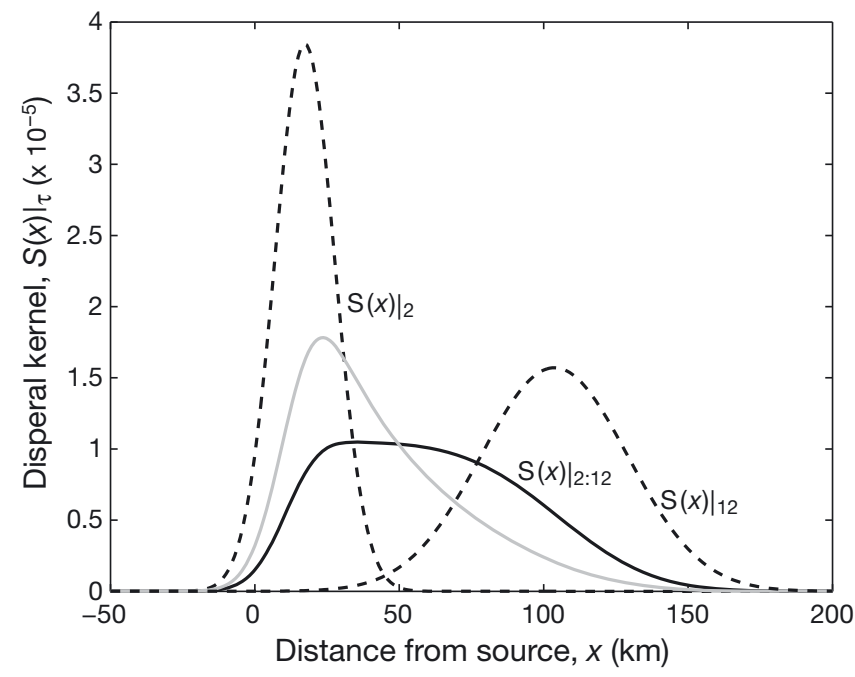

Fig. 1. Dispersal kernels (settlement locations for larvae released at $x=0$ ) for an ocean with mean velocity $u_{0}=0.1 \mathrm{~m}$ $\mathrm{s}^{-1}, \mathrm{SD} u_{1}=0.03 \mathrm{~m} \mathrm{~s}^{-1}$, and Lagrangian timescale $T_{\mathrm{L}}=4 \mathrm{~d}$. Kernels $\left.S(x)\right|_{2}$ and $\left.S(x)\right|_{12}$ (dashed lines) for larvae having larval periods of 2 and $12 \mathrm{~d}$, respectively. Kernels $\left.S(x)\right|_{2: 12}$ (see Eq. 6) for larvae having a competency period of 2 to 12 d. Estimates of $\left.S(x)\right|_{2: 12}$ calculated for uniform settling rate (black line), and for a rate largely determined by exponential mortality (gray line). Kernels are normalised to unit area

Fig. 2 shows spatial kernels calculated for the same ocean conditions for 3 benthic species: red snapping shrimp Alpheus immaculatus (larval period of 7 to 14 d; Shanks et al. 2003), grapsid crab Hemigrapsus penicillatus (15 to $55 \mathrm{~d}_{i}$ Shanks et al. 2003) and red rock lobster Jasus edwardsii (12 to $24 \mathrm{mo}$; Booth
1994). As in Fig. 1, kernels are shown for uniform settling and for high mortality over the larval period. Also shown are Gaussian spatial kernels, $S(x)_{\bar{T}}$ computed using the mean larval period. Coefficients of fit, cof, are given for each spatial kernel $\left.S(x)\right|_{T_{1}: T_{2}}$ where cof is defined by the normalised SD of the difference from the 'mean' kernel:

$$
\text { cof }=\mathrm{SD}\left(\left.S(x)\right|_{T_{1}: T_{2}}-S(x) \tilde{T}\right) / \mathrm{SD}\left(\left.S(x)\right|_{T_{1}: T_{2}}\right)
$$

Fig. 2 illustrates that there is a wide range in how well a Gaussian distribution approximates the spatial kernels. Spatial kernels for Alpheus immaculatus are similar to the estimate made using the mean period $(10.5 \mathrm{~d})$, regardless of mortality, with coefficients of fit of $\sim 0.3$ to 0.5. Kernels for Hemigrapsus penicillatus and Jasus edwardsii have coefficients of fit of 1.4 and 2.7, respectively, and illustrate that a Gaussian kernel is inadequate for these species. For a large range in larval period, the kernel approaches a uniform distribution.

I investigated how the kernel distribution varies with larval period and ocean parameters, assuming uniform settling ( $\gamma=\gamma_{0}$ in Eq. 6 ) for a wide range of ocean conditions $\left(u_{0}\right.$ ranging from 0 to $0.2 \mathrm{~m} \mathrm{~s}^{-1}, u_{1}$ ranging from 0.05 to $0.2 \mathrm{~m} \mathrm{~s}^{-1}$ ) and mean larval periods from 1 to $100 \mathrm{~d}$, with competency periods of 1 to 100 d. Fig. 3 shows cof plotted as a function of non-dimensional competency period, $\Delta \widetilde{T}=\left(T_{2}-T_{1}\right) /$ $\left[\left(T_{2}+T_{1}\right) / 2\right]$, and the ratio of mean to eddy ocean speed, $u_{0} / u_{1}$, for 2 values of $T_{1}(1$ and $10 \mathrm{~d})$. The surfaces of cof are discrete, and functions of $T_{1}$. Fig. 4
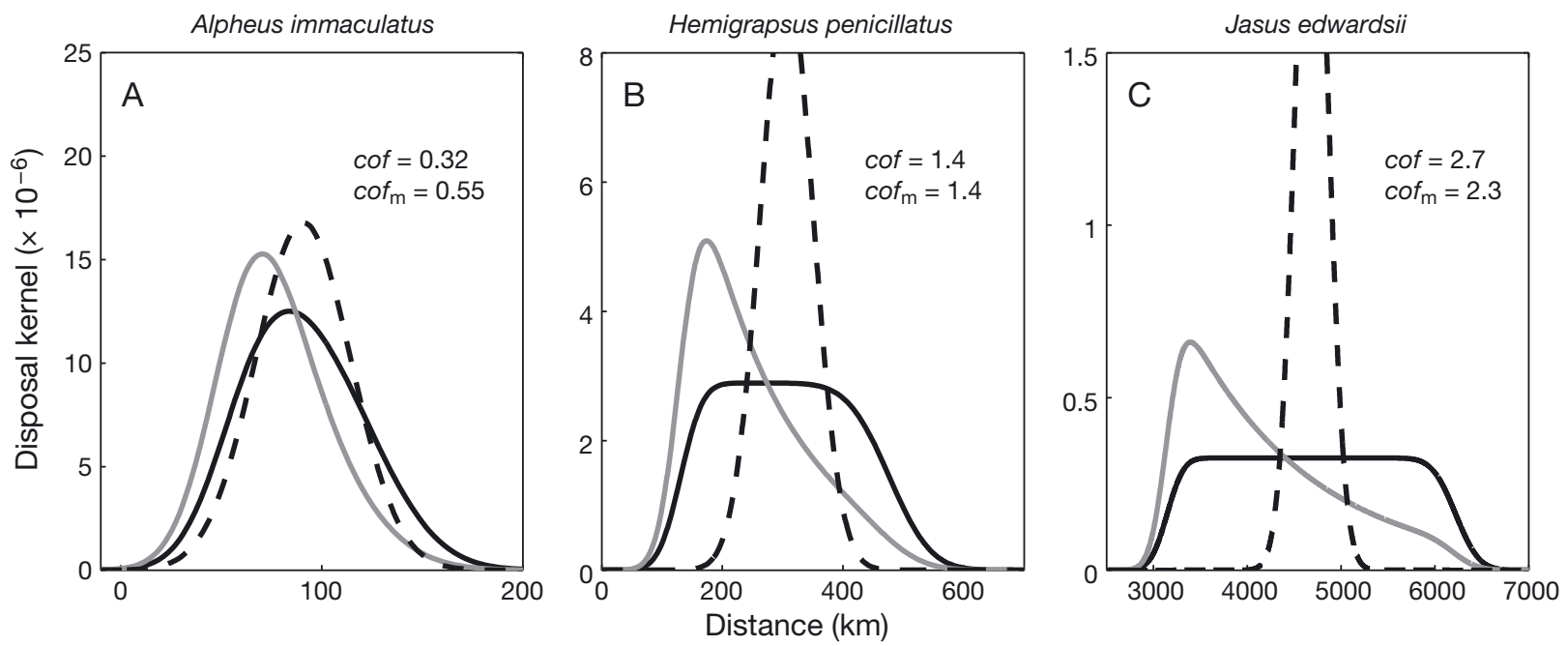

Fig. 2. Larval dispersal kernels $\left.S(x)\right|_{T_{1}: T_{2}}$ calculated for an ocean with mean velocity $u_{0}=0.1 \mathrm{~m} \mathrm{~s}^{-1}, \mathrm{SD}_{1}=0.03 \mathrm{~m} \mathrm{~s}^{-1}$, and Lagrangian timescale $T_{\mathrm{L}}=4 \mathrm{~d}$, for (A) red snapping shrimp Alpheus immaculatus (larval competency period $T_{1}=7$ to $T_{2}=14 \mathrm{~d}$ ), (B) grapsid crab Hemigrapsus penicillatus (15 to $55 \mathrm{~d}$ ); and (C) red rock lobster Jasus edwardsii (12 to 24 mo). The 2 estimates of $\left.S(x)\right|_{T_{1}: T_{2}}$ for each species were calculated for no larval mortality (black line) and exponential mortality (gray line). Dashed lines are kernels, $S(x)_{\bar{T}}$, based on the mean larval period. Coefficients of fit, cof, are the normalised SD of difference between $\left.S(x)\right|_{T_{1}: T_{2}}$ and $S(x)_{\bar{T}}$, where $\mathrm{m}$ denotes kernels calculated assuming larval mortality 

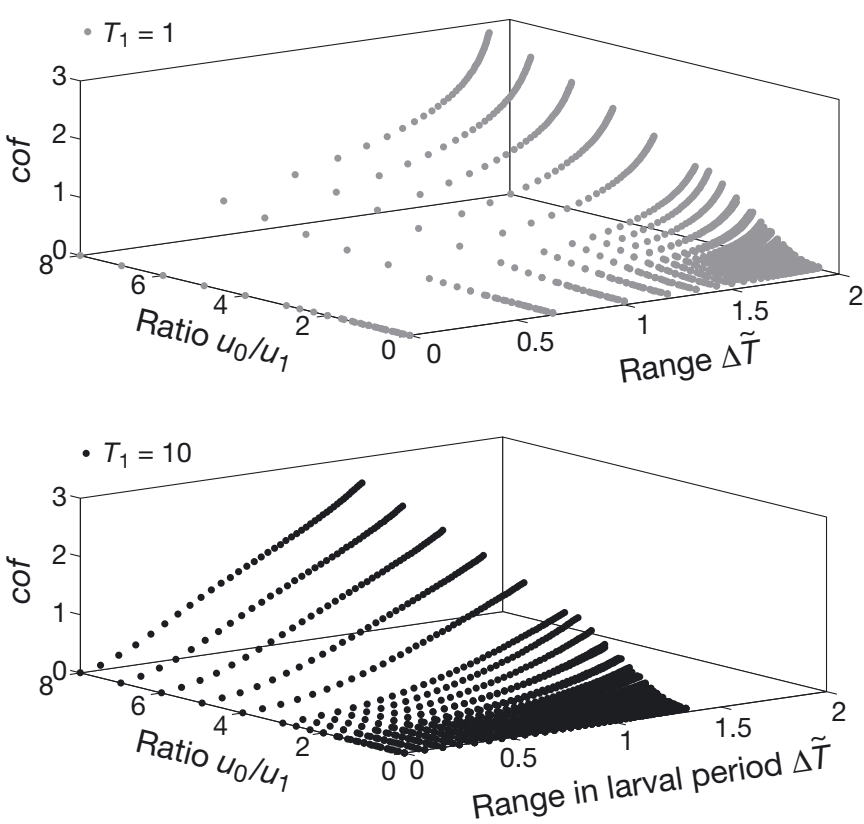

Fig. 3. Coefficient of fit cof, between spatial kernels $\left.S(x)\right|_{T_{1}: T_{2}}$ and $S(x)_{\bar{T}}$ (computed assuming no larval mortality). Coefficients are plotted as a function of normalised larval competency period range $\Delta \widetilde{T}=\left(T_{2}-T_{1}\right) /\left(T_{2}+T_{1}\right)$, and ratio of mean to $\mathrm{SD}$ of

flow $u_{0} / u_{1}$, for 2 different values of minimum larval period

shows cof in more detail for $T_{1}=10 \mathrm{~d}$, contoured as a function of the competency period $\left(T_{2}-T_{1}\right)$ and the ratio $u_{0} / u_{1}$. The kernels become less Gaussian as the minimum larval period, $T_{1}$, or the range, $\Delta \widetilde{T}$, increase.

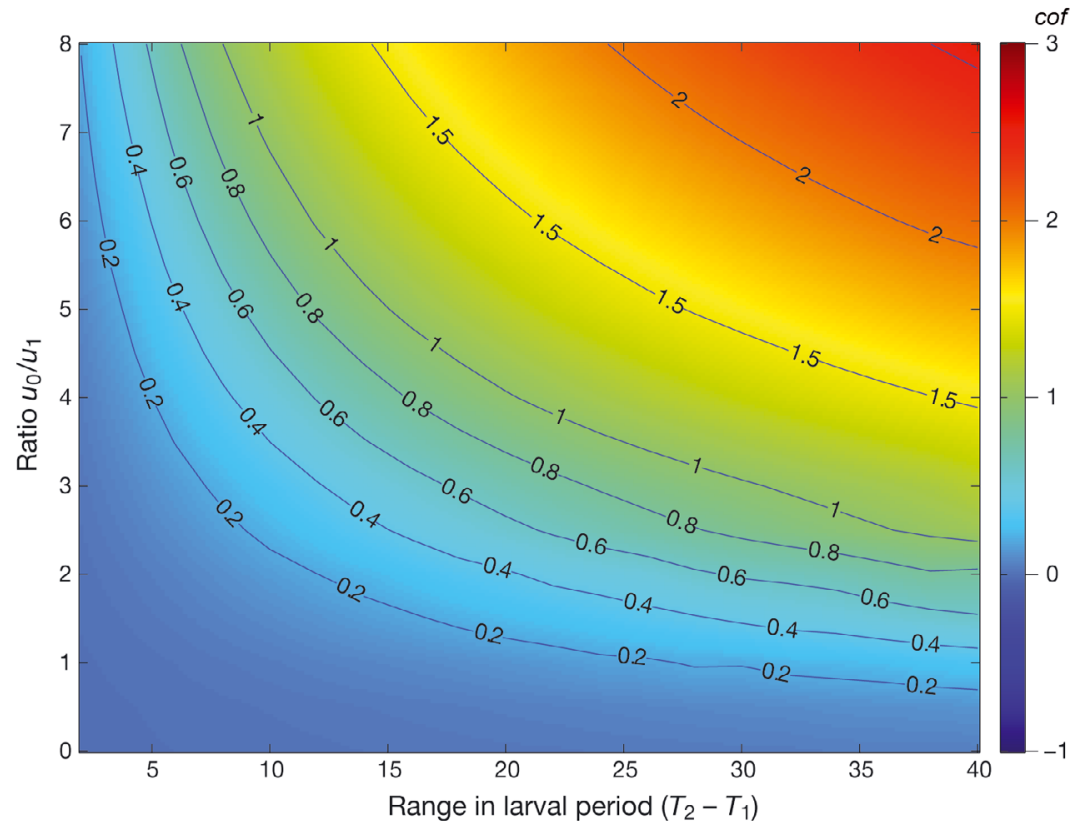

Fig. 4. Coefficient of fit cof, between spatial kernels $\left.S(x)\right|_{T_{1}: T_{2}}$ and $\left.S(x)\right|_{\vec{T}}$ (computed assuming no larval mortality) for minimum larval period $T_{1}=10 \mathrm{~d}$ (Fig. 3b). The coefficient is contoured as function of range in competency period $\left(T_{2}-T_{1}\right)$, and ratio of mean to $\mathrm{SD}$ of eddy flow $u_{0} / u_{1}$

The kernels also become progressively less Gaussian as the ratio of mean to eddy flow $\left(u_{0} / u_{1}\right)$ increases. Since the eddy diffusivity depends on the strength of the eddy field (Eq. 2), this demonstrates that the kernels become less Gaussian as $K$ decreases.

In principle, one could use some a priori criterion to choose a critical value of cof and determine whether estimating the spatial kernel from the mean larval period is acceptable. Based on Fig. 2, this critical value of cof is about 0.2 , and Fig. 4 shows that for $T_{1}=$ $10 \mathrm{~d}$, when the mean flow is small compared to the eddy variability, the estimate of the kernel using the mean larval period is acceptable for a wide range of larval periods. However, when the eddy variability in the ocean is small, compared to the mean flow, the kernel becomes significantly non-Gaussian even for short competency periods.

\section{DISCUSSION}

Many common species have larval competency periods in the 1 to $60 \mathrm{~d}$ range (Shanks et al. 2003), and Fig. 2 illustrates that for some realistic ocean conditions and competency periods, the spatial kernel is likely to be sufficiently non-Gaussian to warrant explicitly calculating it over the range of larval periods. As an example, the SD of the kernel for Hemigrapsus penicillatus is $108 \mathrm{~km}$ when calculated tency period, compared to $43 \mathrm{~km}$ when calculated using the mean period.

Sensitivity analysis as illustrated in Figs. 3 \& 4 can determine whether a Gaussian approximation is adequate for any given problem. However, Eq. (6) is easy to compute, and in general it is not necessary to map cof in parameter space. The sensitivity analysis has been done here mainly to show that kernels become less Gaussian as either the mean larval period or the range in periods increase, or if the eddy variability decreases. This last point is perhaps counter-intuitive because one might expect kernels to become more nonGaussian as the ocean becomes more dispersive (in fact, when all other variables are held constant, as $K$ increases, the spread in $\left.S(x)\right|_{T_{1}: T_{2}}$ increases, but the kernel becomes more Gaussian).

Figs. $3 \& 4$ show that in some cases, particularly when eddy variability is 
small compared to mean current velocity, there needs only to be a few days range in the competency period before the spatial kernel becomes significantly non-Gaussian.

The present study has treated age distribution in larval settlement ( $\gamma$ in Eq. 6) with 2 simple functions because of the difficulty of describing a general function for mortality and age-dependent metamorphosis. In some cases, it may be that larval mortality is so high that there are very few late settlers and one can reasonably compute spatial kernels using only the earliest settlers. But in other cases, seasonality in metamorphosis from the larval to post-larval stages may well outweigh mortality in the later settlers. For example, Booth (2002) shows that New Zealandwide settlement of Jasus edwardsii occurs steadily over several months in autumn and early winter, but then peaks during the following 3 months.

This study has treated the ocean with a simple 1-dimensional model having homogeneous statistics. In particular, the eddy diffusivity has been assumed to be constant. In reality, both the Eulerian mean flow and eddy variability will be spatially variable, so that the Lagrangian velocity statistics are heterogeneous. The net result is that eddy diffusivity becomes scale dependent, and this is reflected in observations of $K$ that vary from $10^{-1} \mathrm{~m}^{2} \mathrm{~s}^{-1}$ for scales of about $10 \mathrm{~m}$ (e.g. Bogucki et al. 2005) to $10^{4} \mathrm{~m}^{2} \mathrm{~s}^{-1}$ for the tropical open ocean (e.g. Bauer et al. 2002). The value of $K$ used here $\left(300 \mathrm{~m}^{2} \mathrm{~s}^{-1}\right)$ is typical for the continental shelf (e.g. Li \& Cai 2011, Stachelhaus et al. 2012). From a larval dispersal perspective, if the diffusivity increases significantly during larval competency, kernels are likely to be slightly more Gaussian than shown here.

Non-Gaussian kernels may have causes other than a long competency period. For example, in a study of dispersal along the Chilean coast using realistic coastline and numerical models and a single 30 d larval period, Aiken et al. (2007) found considerable departure from the expected Gaussian dispersal kernels leading to 'significant variation in the spatial pattern of connectivity among local sites, with some acting as net sources and some as net sinks'. The likely causes of these non-Gaussian distributions are spatial variations in the Eulerian flows (both mean and eddy variability). The results presented here show that the locations of sources and sinks determined by Aiken et al. (2007) could well depend on the larval period; if that is so, interpretations of connectivity that are based on a single value for the larval period will be misleading.
For many species, spatial kernels will be different when calculated using the full competency period instead of the mean larval period. Prior to numerical simulations, Eq. (6) can be used to determine whether using a realistic range in larval periods is necessary. Lagrangian studies (individual based modelling) are well suited to such an approach by compiling settlement locations over the range of larval periods.

Acknowledgements. I thank David Bowden for discussions that inspired this study, and Graham Rickard for critical comments. This study was funded by the Foundation for Research, Science and Technology, New Zealand, Contract Nos. C01X0223 and C01X0501.

\section{LITERATURE CITED}

Aiken CM, Navarrete SA, Castillo MI, Castilla JC (2007) Along-shore larval dispersal kernels in a numerical ocean model of the central Chilean coast. Mar Ecol Prog Ser 339:13-24

Bauer S, Swenson MS, Griffa A (2002) Eddy mean flow decomposition and eddy diffusivity estimates in the tropical Pacific Ocean. 2. Results. J Geophys Res 107:3154 doi 10.1029/2000JC000613

Bogucki DJ, Jones BH, Carr ME (2005) Remote measurements of horizontal eddy diffusivity. J Atmos Ocean Technol 22:1373-1380

Booth JD (1994) Jasus edwardsii larval recruitment off the east coast of New Zealand. Crustaceana 66:295-317

Booth JD (2002) Early life history, recruitment processes and settlement of spiny lobsters. Fish Sci 68:384-389

> Chiswell SM (1996) Variability in the Southland Current, New Zealand. NZ J Mar Freshw Res 30:1-18

> Chiswell SM (2011) Temporal kernels of island connectivity: a generalised approach. Aquat Biol 12:205-214

> Cowen RK, Sponaugle S (2009) Larval dispersal and marine population connectivity. Annu Rev Mar Sci 1:443-466

Kinlan BP, Gaines SD, Lester SE (2005) Propagule dispersal and the scales of marine community process. Divers Distrib 11:139-148

Largier JL (2003) Considerations in estimating larval dispersal distances from oceanographic data. Ecol Appl 13: 71-89

Li C, Cai WJ (2011) On the calculation of eddy diffusivity in the shelf water from radium isotopes: high sensitivity to advection. J Mar Syst 86:28-33

Mitarai S, Siegel DA, Winters KB (2008) A numerical study of stochastic larval settlement in the California Current system. J Mar Syst 69:295-309

O'Connor MI, Bruno JF, Gaines SD, Halpern BS, Lester SE, Kinlan BP, Weiss JM (2007) Temperature control of larval dispersal and the implications for marine ecology, evolution, and conservation. Proc Natl Acad Sci USA 104: 1266-1271

Rupolo V (2007) Observing turbulence regimes and Lagrangian dispersal properties in the oceans. In: Griffin DA, Kirwin AD, Mariano AJ, Ozgokmen TM, Rossby R (eds) Lagrangian analysis and prediction of coastal and ocean dynamics. Cambridge University Press, Cambridge, 


$$
\text { p 231-274 }
$$

Shanks AL, Grantham BA, Carr MH (2003) Propagule dispersal distance and the size and spacing of marine reserves. Ecol Appl 13:159-169

> Stachelhaus SL, Moran SB, Ullman DS, Kelly RP (2012) Cross-shelf mixing and mid-shelf front dynamics in the mid-Atlantic Bight evaluated using the radium quartet.

Editorial responsibility: Christine Paetzold, Oldendorf/Luhe, Germany
J Mar Res 70:141-172

Trakhtenbrot A, Nathan R, Perry G, Richardson DM (2005) The importance of long-distance dispersal in biodiversity conservation. Divers Distrib 11:173-181

Walker MM (1984) Larval life span, larval settlement, and early growth of Evechinus chloroticus (Valenciennes). NZ J Mar Freshw Res 18:393-397

Submitted: January 11, 2012; Accepted: July 30, 2012

Proofs received from author(s): August 26, 2012 